\title{
Research Paper \\ The Effect of Spiritual Self-Care Education on the Care Burden of Mothers With Children Hospitalized in Intensive Care Units for Open Heart Surgery
}

\author{
Maryam Dalir $^{1} \odot{ }^{*}$ Soheila Mashouf ${ }^{1} \odot$, Simin Esmailpourzanjani ${ }^{1}$
}

1. Department of Nursing, School of Nursing and Midwifery, Tehran Medical Branch, Islamic Azad University, Tehran, Iran.

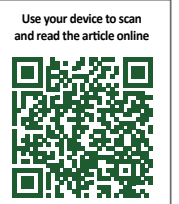

Cttat On: Dalir M, Mashouf S, Esmailpourzanjani S. [The Effect of Spiritual Self-Care Education on the Care Burden of Mothers With Children Hospitalized in Intensive Care Units for Open Heart Surgery (Persian)]. Complementary Medicine Journal. 2020; 10(1):34-45. https://doi.org/10.32598/cmja.10.1.866.1

https://doi.org/10.32598/cmja.10.1.866.1

Keywords:

Spiritual self-care, Care burden, Education, Hospitalization.

\section{A B S TRACT}

Objective Spiritual health of caregivers has association with the patients' health, but less attention has been paid to spiritual self-care education in health programs. This study aimed to assess the effect of spiritual self-care education on the care burden of mothers with children in hospitalized in Intensive Care Units (ICUs) for open heart surgery.

Methods This quasi-experimental study was conducted on 60 mothers of children hospitalized in ICUs of Shahid Modarres Hospital in Tehran, Iran. They were selected randomly from among those gave informed consent to participate in the study. The data collection tools were a demographic form and Zarit Burden Interview which were completed before and after intervention. The spiritual self-care education was presented using the teaching aids in four 45-min sessions, twice a week. Collected data were analyzed in SPSS V. 24 software using descriptive (frequency, percentage) and inferential (paired t-test) statistics at a significance level of $\mathrm{P}<0.05$.

Results Before the intervention, $72.3 \%$ of the samples had a severe care burden and $24.5 \%$ had a very severe care burden, which decreased after the intervention such that only $32.7 \%$ had a severe care burden. The mean and standard deviation of mothers' care burden also decreased after the educational intervention (from $62.3 \pm 7.6$ to $42.1 \pm 3.8$ ). The results of paired t-test showed that this difference was statistically significant $(P<0.001)$.

Conclusion Spiritual self-care education can reduce the care burden of children' caregivers; hence, planning and implementing appropriate programs to increase self-care knowledge of caregivers (especially the mothers of children with heart problems) and medical staff and paying more attention to mothers' education are recommended.

\section{Extended Abstract}

\section{Introduction}

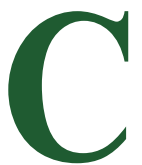

ongenital heart disease is one of the most important disorders and its prevalence is about 8 in 1000 in European countries. Congenital anomalies and congenital heart disease are the leading causes of child mortality in the world [1]. About $1-2 \%$ of babies are usually born with heart problems. According to global statistics, for every 1,000 births in the world, 8-9 babies have congenital heart disease, mild to severe $[1,2]$. Caring for children with heart problems is a major psychological crisis for parents, especially mothers; it causes disability in the child and puts a high stress on the mother [3]. The results of Gray et

\section{* Corresponding Author}

Soheila Mashouf, PhD.

Address: Department of Internal-Surgery Nursing, Tehran Medical Branch, Islamic Azad University, Tehran, Iran.

Tel: +98 (21) 22006660

E-mail: smashouf@iautm.ac.ir 
al.'s showed that mothers with this type of children tolerate higher levels of anxiety and psychological problems than mothers with normal children [3].

Patients with heart problems and their caregivers, due to the consequences of disease and treatment, face with changes in their needs and require self-care to overcome their problems [4]. Adherence to self-care behaviors is important. Self-care education can improve patients' functional abilities and disease processes. One type of self-care is spiritual self-care. According to studies, spiritual self-care may affect maternal care performance due to the lack of education in mothers prior to and during the care period. Studies emphasizes that in order for the mother to be able to understand the patient's spirituality and meet her spiritual needs, it is necessary to determine and expand the mothers' self-care knowledge $[5,6]$. In this regard, this study aimed to determine the effect of spiritual self-care education on the care burden of mothers of children hospitalized in Intensive Care Units (ICUs) for open heart surgery

\section{Materials and Methods}

This quasi-experimental study was performed on 60 mothers of children hospitalized in ICUs for open heart surgery in a hospital affiliated to Shahid Beheshti University of Medical Sciences. After explaining the study method and obtaining informed consent from all of them, they were asked to complete a demographic form and Zarit Burden
Interview (ZBI). In the next step, the spiritual self-care education was presented through slide show, movie, and booklet for two weeks, four sessions each for 45 minutes. The sessions were held in the presence of the mothers and with the title of "helping yourself and the child with self-care education". After one month, the questionnaires were completed again by the mothers and the information was collected. They were analyzed in SPSS V. 24 software using descriptive (frequency, percentage) and inferential (paired $\mathrm{t}$-test) statistics at a significance level of $\mathrm{P}<0.05$.

\section{Results}

The majority of participants (40\%) had age between $36-$ 45 years; 93\% were married; $44.2 \%$ were employed; $41 \%$ had a high school diploma; $73.8 \%$ had monthly income between 1-2 million Tomans; $60.2 \%$ were living in their own house; $67.2 \%$ had $2-3$ children and $47.2 \%$ had 3 children; and $100 \%$ had no history of childcare in cardiac ICUs. Before the intervention, $24.5 \%$ of mothers had very severe, $72.3 \%$ severe, and 3.2\% had moderate care burden. After the intervention, this rate changed and only $32.5 \%$ had a severe care burden and $57.7 \%$ had a moderate care burden (Table 1). The Mean \pm SD of mothers' care burden score before and after educational intervention were $62.3 \pm 7.6$ and $42.1 \pm 3.8$, respectively which indicates a significant decrease after the intervention. The results of the t-test showed a statistically significant difference between the mean pretest and posttest scores of care burden $(\mathrm{P}<0.001)$ (Table 2$)$.

Table 1. The statistics of care burden in mothers before and after intervention based on its severity

\begin{tabular}{ccc}
\hline \multirow{2}{*}{ Care Burden } & \multicolumn{2}{c}{ No. (\%) } \\
\cline { 2 - 3 } & Pre-test & Post-test \\
\hline Low (0-20) & $0(0)$ & $6(9.8)$ \\
\hline Moderate (21-40) & $2(3.2)$ & $35(57.5)$ \\
\hline Severe (41-60) & $44(72.3)$ & $20(32.7)$ \\
\hline Very severe (61-88) & $15(24.5)$ & $0(0)$ \\
\hline Total & $61(100)$ & $61(100)$ \\
\hline
\end{tabular}

Table 2. Comparing the mean of care burden in mothers before and after intervention

\begin{tabular}{cccc}
\hline Stage & Mean \pm SD & t & P \\
\hline Before intervention & $62.3 \pm 7.6$ & & $\leq 0.001$. \\
After intervention & & 39.98 & \\
\end{tabular}




\section{Discussion}

The results showed that $72.3 \%$ of mothers had a severe care burden before the intervention (62.3 \pm 7.6$)$. Khalifehzadeh et al. [7] in a study reported a moderate level of maternal care burden before the intervention (55.5 \pm 10.4$)$, which was lower and better than the mean value reported in our study. Haghgoo et al. [8] also reported the moderate burden on family caregivers of patients with mental disorders. The reasons for the high level of care burden in the present study can be the low literacy, occupation (worker), and lack of caring for children with heart problems in the majority of the study samples and most importantly, a mother cannot adapt quickly to the birth of a child with congenital heart disease. Being in such a situation creates a heavy care burden in a mother.

Spore [9] examined care burden of mothers in two control and intervention groups. His results showed that the mother cannot spend enough time with the family due to the high care needs of her sick child, which causes a high care burden. In his study, the average maternal care burden was between 35 and 45. This discrepancy can be due to the appropriate approaches to welfare and social services in the developed countries where both the government and the family members free the mothers from other tasks and responsibilities to take care of the child with all her power and time.

Heydari et al. [10] in studying the effect of family-centered care on the burden of family caregivers in patients with epilepsy showed that the mean of care burden in the caregivers before the intervention was $37.24 \pm 13.5$, which is in the moderate level and is not consistent with our result. It can be due to the type of disease. Epilepsy is a chronic and transient disease and mothers are often the primary caregivers of these patients at home; the risk of dying in epileptic patients is lower than in patients with congenital heart disease. Talebi et al. [11] in examining the effect of mindfulness-based education on the care burden and psychological capital of the parents of children with attention deficit hyperactivity disorder reported their severe care burden (93.46 \pm 61.15$)$ before the intervention which is in agreement with our result.

After determining the amount of care burden and in order to improve its condition, educational intervention was performed for spiritual self-care. After the intervention, their burden was reduced from $62.3 \pm 7.6$ to $42.1 \pm 3.8$, and most of mothers $(57.7 \%)$ showed a moderate care burden. Consistent with this result, Amakali et al. [12] in a study on 65 rural parents of children with heart diseases in Namibia who received the empowerment strategies for dealing with feelings of inability to care during 6 sessions showed that the ability to manage problems enables parents to provide quality care and facilitate optimal performance for children. Our result is also consistent with the results of Khalifehzadeh et al. [7] who showed that the amount of care burden after training was significantly reduced.

\section{Conclusion}

Spiritual self-care education can reduce the care burden of mothers with children hospitalized in ICUs for open heart surgery. It can prepare mothers for the role of caregiver and proper management of the care process.

\section{Ethical Considerations}

\section{Compliance with ethical guidelines}

This article ethically approved by the ethics Committee (Code: IR.IAU.TMU.REC.1397.082).

\section{Funding}

This article has extracted from the Master thesis of the first author, Department of Nursing, School of Nursing and Midwifery, Tehran Medical Branch, Islamic Azad University.

\section{Authors' contributions}

All authors contributed equally in preparing this paper.

\section{Conflicts of interest}

The authors declare no conflict of interest

\section{Acknowledgements}

The authors would like to thank staffs and administration of Islamic Azad University for their cooperation and providing data for conducting this research. 


\title{
تأثير آموزش خودمراقبتى معنوى بر بار مراقبتى مادران كودكان بسترى در بخش هاى مراقبتهاى ويزه جراحى قلب باز
}

\author{
مريم دلير' ه. "سهيلا مشعوف' 0، سيمين اسماعيليور زنجانى' \\ ا. كروه يرستارى، دانشكله يرستارى و مامايى، واحد علوميزشكى تهران، دانشكاه آزاد اسلامى، تهاني، ايران.
}

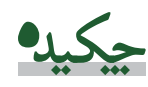

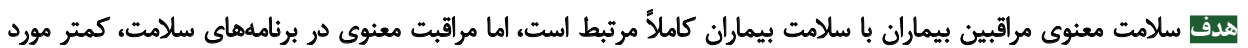

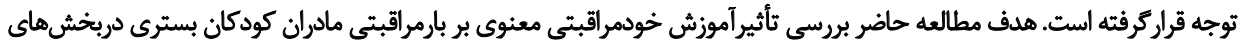

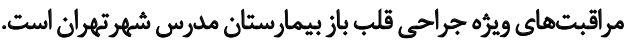

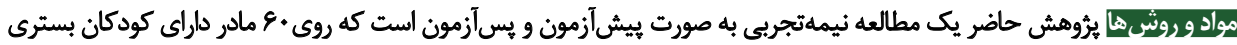

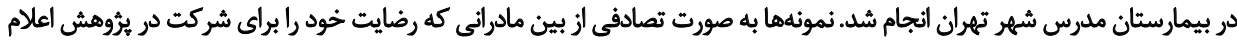

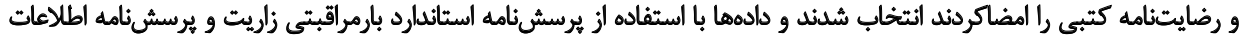

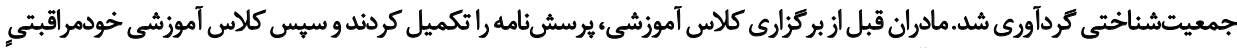

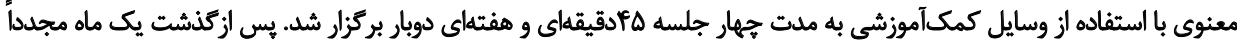

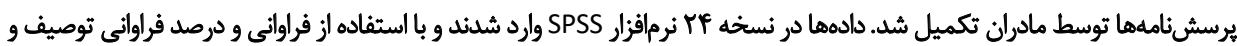

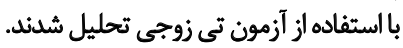

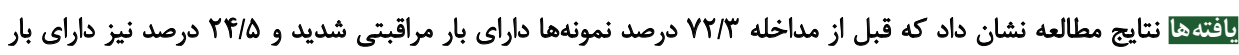

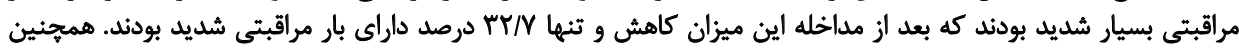

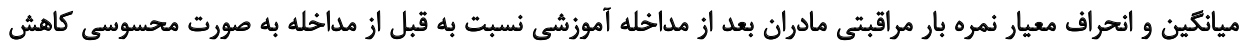

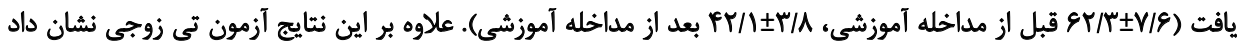

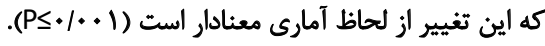

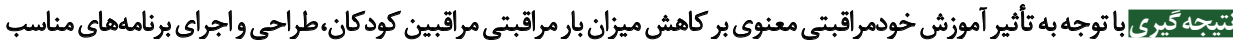

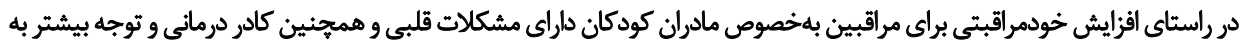

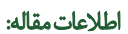

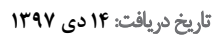

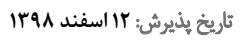

يك بحران روحى بزرى محسوب مىشود و براى كودى ناتوانى رئى

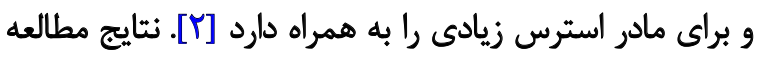

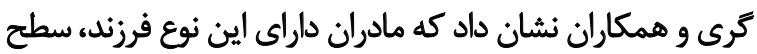

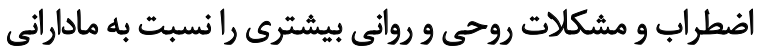

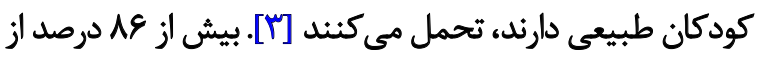

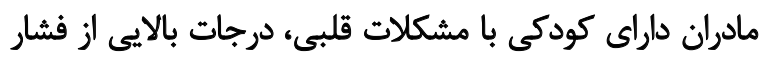

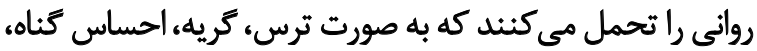

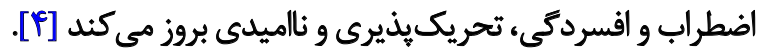
در دهdهاى اخير مطالعات زيادى روى عوامل خطرى كه

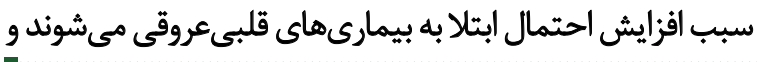

بيمارىهاى قلبى مادرزادى يكى از ناهنجارىهاى مهرم است

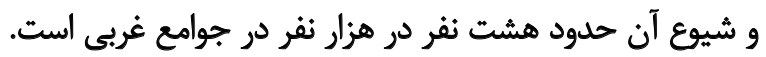

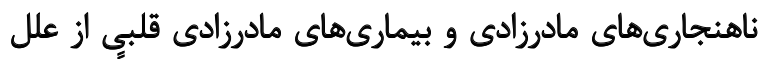

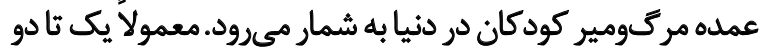

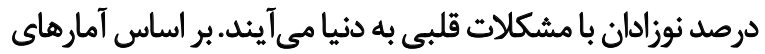

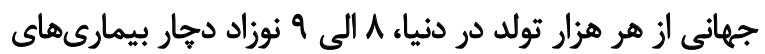

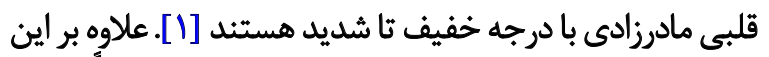
مراقبت از كودكان با مشكلات قلبى براي والدين، خصوصاً مادران

تويسئده مسئول: توينال:

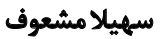
نشانى: تهران، دانشكاه آزاداد اسلامى، واحد علوميزشكى تهران، داتشكده يرستارى و مامايى، كروه يرستارى.

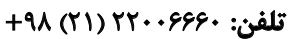
يست الكترونيكى: smashouf@iautm.ac.ir 
اين در حالى است كه آنان بايد به رفع نيازهاى خانواده نيز بيردازند

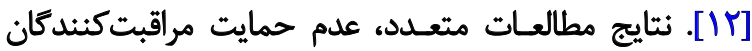

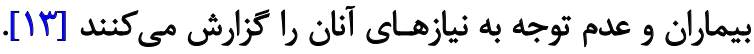

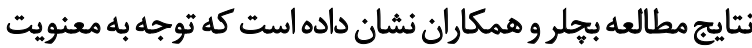

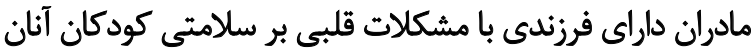

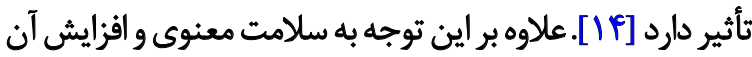

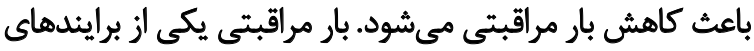

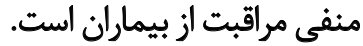

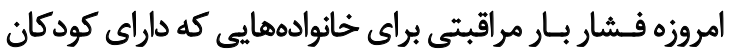

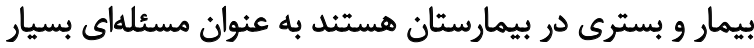

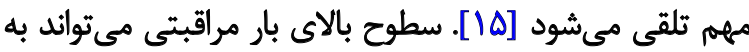

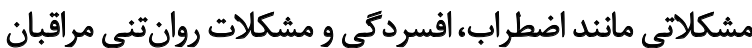

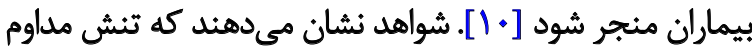

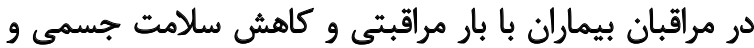

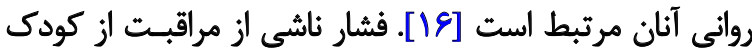

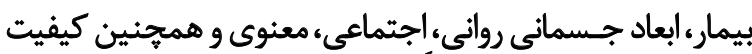

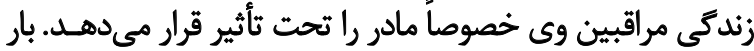

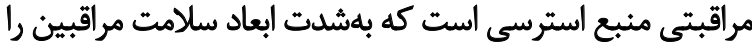

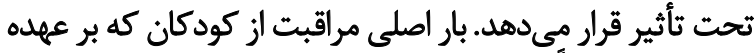

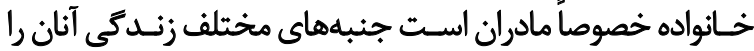
تحت تأثير قرار مى دهد [IV] [IV]

بار مراقبتى مادران داراي كودك بيمار در سطوح بسيار بالايى قرار

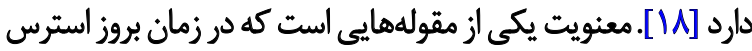

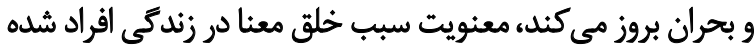

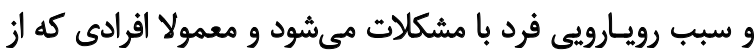

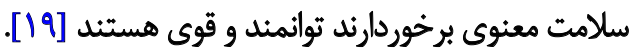

بر اساس شواهد موجود مراقبت معنوى ممكن است به علت

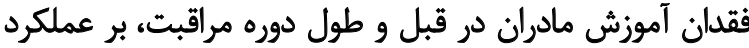

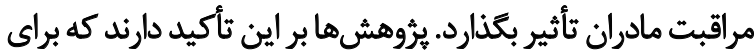

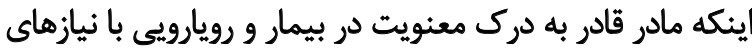

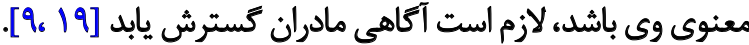

با توجه به نقش غيرقابل انكار مراقبت معنوى در سلامت بيماران

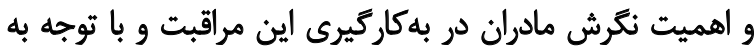

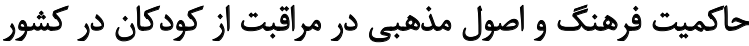

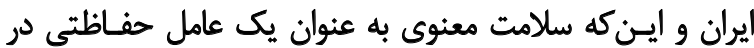

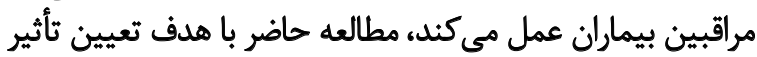

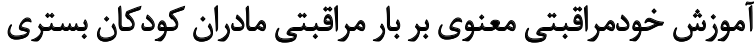

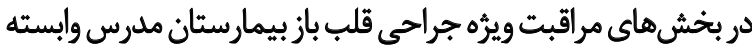

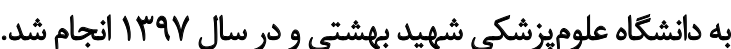

همجنين عوامل محافظتى، صورت كرفته است. آكاهى از عوامل

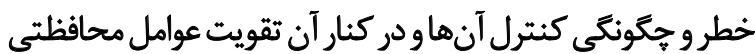

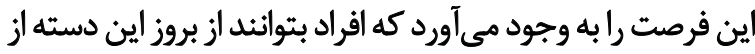

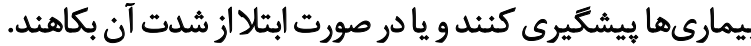

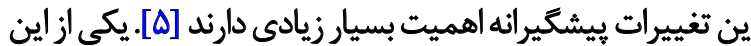
عوامل محافظتى، بحث بهبود خودمراقبتئي است.

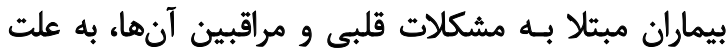

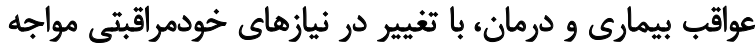

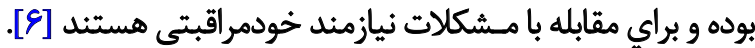

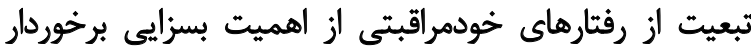

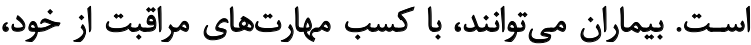

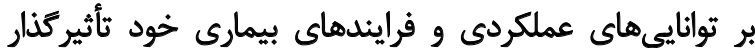

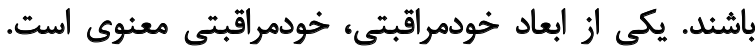

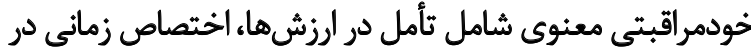

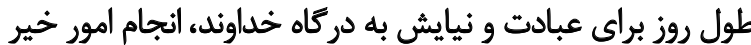

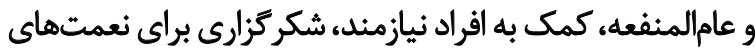

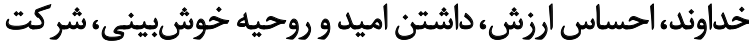

در مراسم دينى، مطالعه كتب مذهبى و معنوى مى شيدود [V]

وجود معنويت براى والدين و مراقبت كنئدكان كودكان داراى

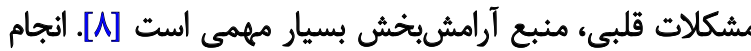

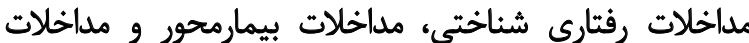

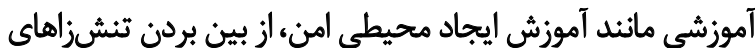

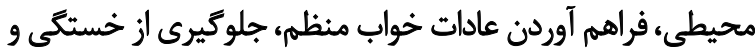

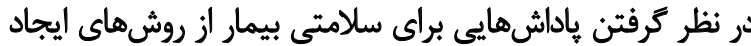

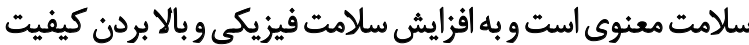

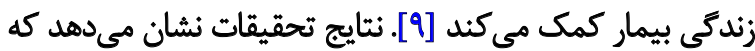

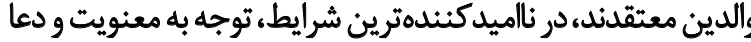

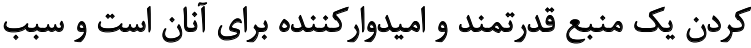

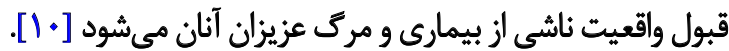

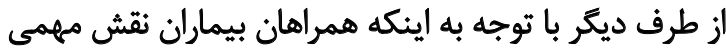

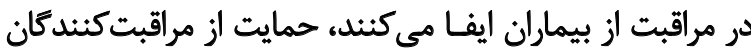

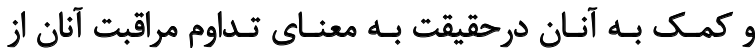

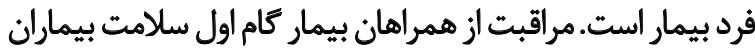

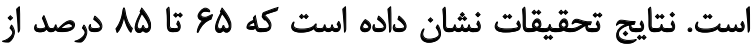

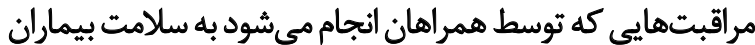

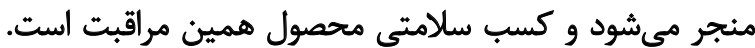

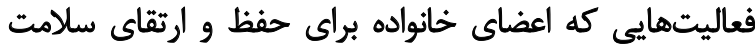

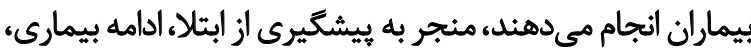

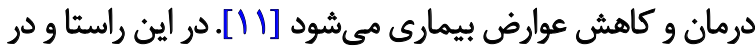

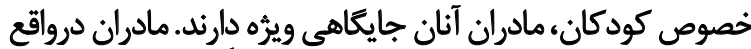

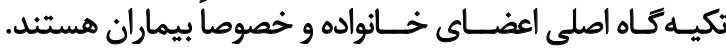

نتايج مطالعات نشان دادهاند كه در شرايط بيمارى و خصوصاً

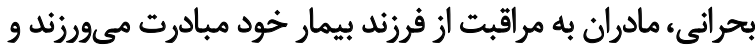


كرونباخ در مطالعه حاضر /Ar • به دست آمد كه بيانكر قابل قبول

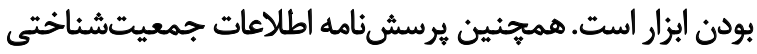

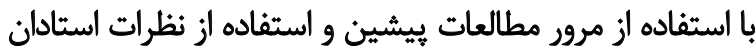

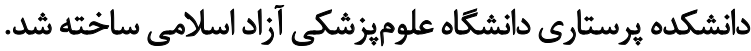

اين تحقيق حاصل كار بايايانامه دانشجويى مربوط به دانشكده

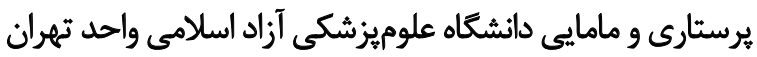

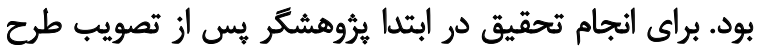

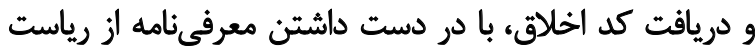

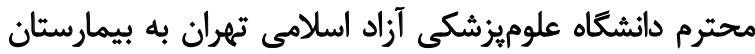

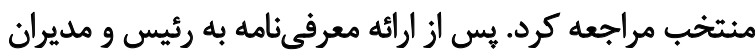

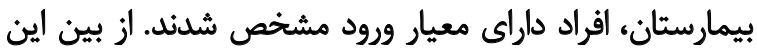

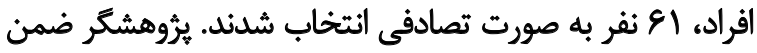

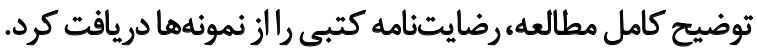

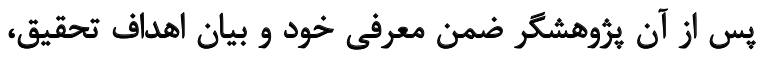

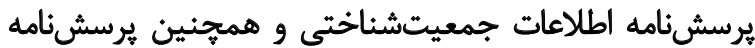
زاريت را به آنان داد تا ير كنئد.

يس از آن اطلاعات جمعآورى شد و در مرحله بعد كلاس

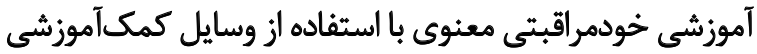

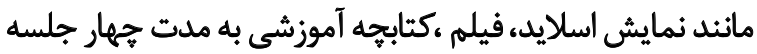

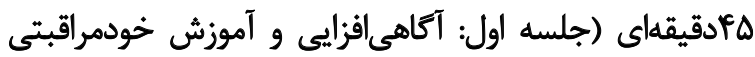

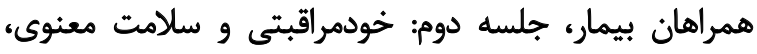

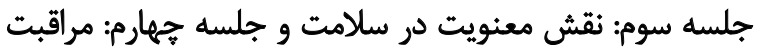

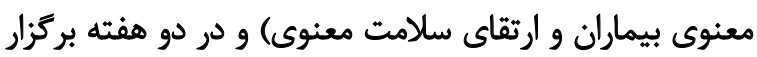

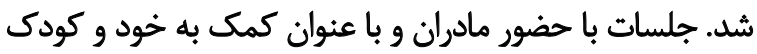

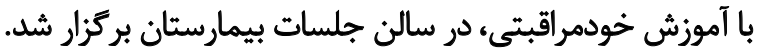

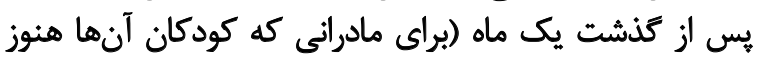

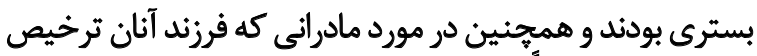

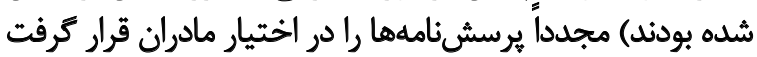

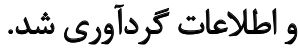

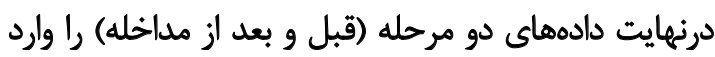

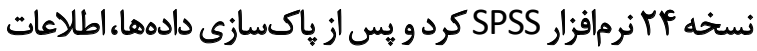

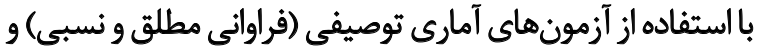
استنباطى (آزمون تى زوجى) مورد تحليل قرار ترفيتند.

يافتهها

نتايج يُؤهش حاضر نشان داد كه اكثريت واحدهاى

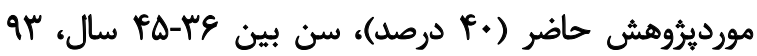

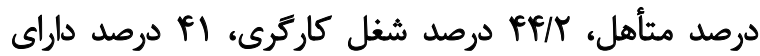

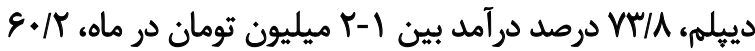

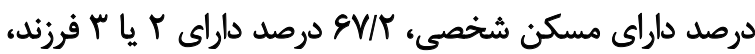

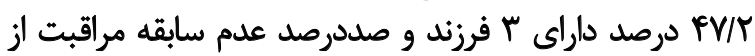

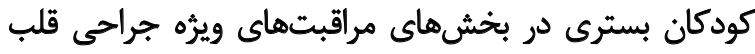
داشتند (جدول شماره ()).

\section{مواد و روشها}

اين يرُوهش از نوع نيمهتجربى و محيط مطالعه بيمارستان

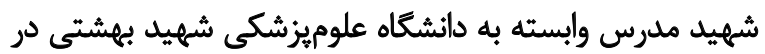

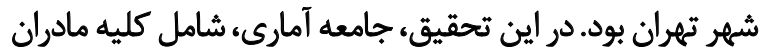

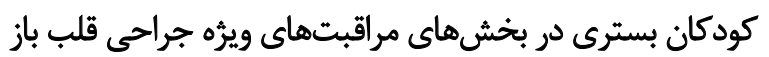

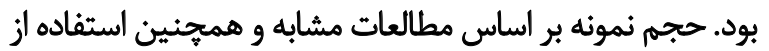

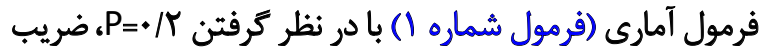

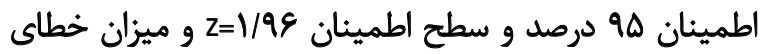

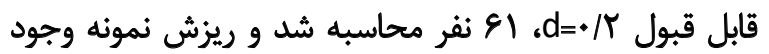

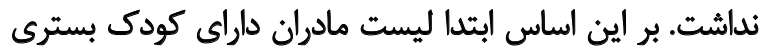

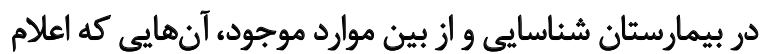

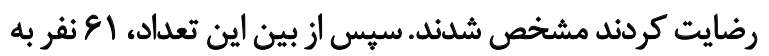

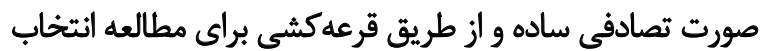

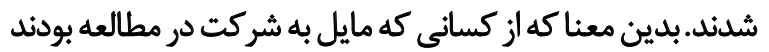

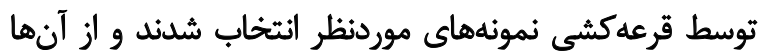

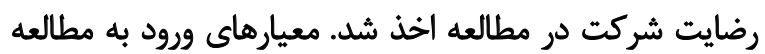

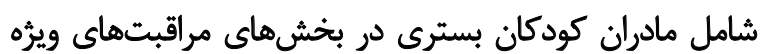

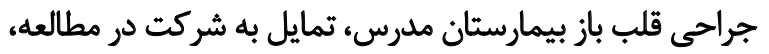

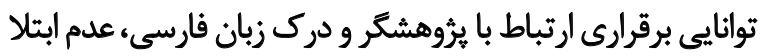

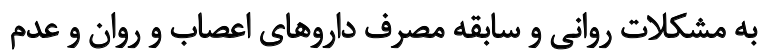

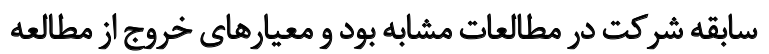

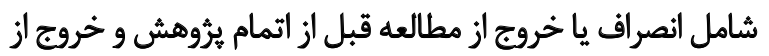

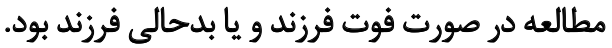

1. $n=\frac{z^{2} p(1-p)}{d^{2}}$

ابزار كردآورى اطلاعات در اين تحقيق يرسشنامنامه اطلاعات

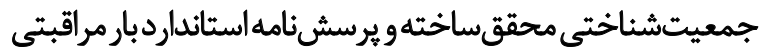

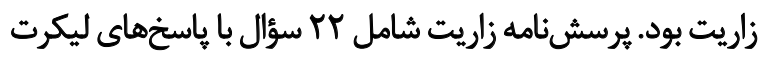

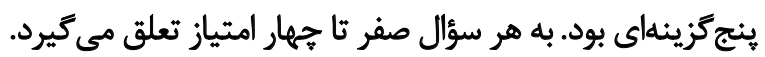

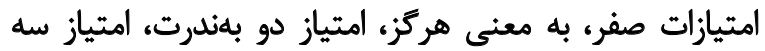

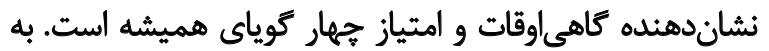

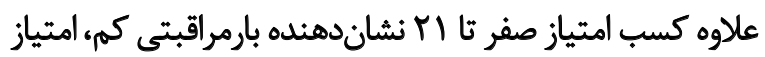

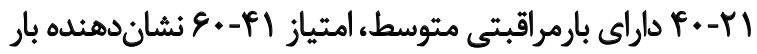

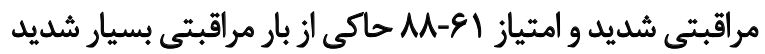

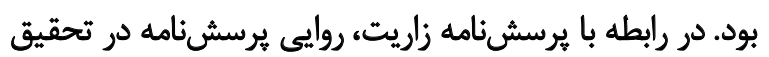

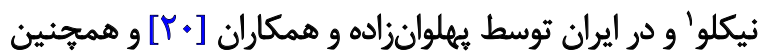

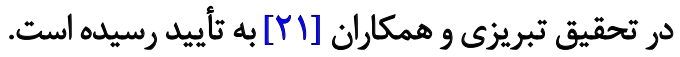

هايايى اين يرسش آنامه در تحقيق يهلوانز ادهو همكاران از طريق

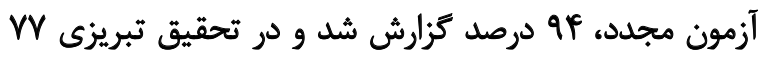

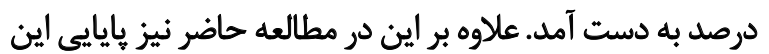

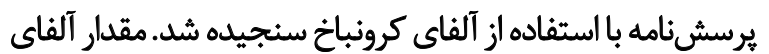


جدول ا. توزيع فراوانى مطلق و نسبى متغيرهاى جمعيتشئاختى

\begin{tabular}{|c|c|c|c|}
\hline فراوافي نسبي & فراواني مطلق & & \\
\hline$\pi / N$ & r. & ها-+r سال & \multirow{4}{*}{ سن } \\
\hline$r e / r$ & 18 & Pro-ro & \\
\hline$r .1$. & TA & ع rata wlل & \\
\hline - & - & بالاى هt سال & \\
\hline ar & $\Delta$ & مثأهل & \multirow{2}{*}{ تأهل } \\
\hline$A$ & $\Delta$ & هنتاركه & \\
\hline - & - & بيكار & \multirow{4}{*}{ اشغل } \\
\hline Pr/T & $r$ & كار5 & \\
\hline refr & 18 & كارمند & \\
\hline rag & M & شغل آزاد & \\
\hline 198 & ir & ابتدائى & \multirow{5}{*}{ تحصيلات } \\
\hline $18 / F$ & 1. & راهنمايى & \\
\hline Fl. & ra & دييلم & \\
\hline r & If & فوق دييلم & \\
\hline • & • & ليساتس & \\
\hline$V^{2} / \Lambda$ & fo & r-1 ميليون تومان & \multirow{5}{*}{ ميزان درآمد } \\
\hline $9 / \wedge$ & 8 & r-r ميليون تومان & \\
\hline $\mid \& / f$ & 1. & با-" ميليون تومان & \\
\hline + & - & ه-r ميليون تومان & \\
\hline + & + & بالاى ه ميليون تومان & \\
\hline$r m / r$ & if & اجارياى & \multirow{3}{*}{ نوع مسكن } \\
\hline $8 \cdot / r$ & $r$ & شخصى & \\
\hline $18 / 0$ & 1. & سازمانى & \\
\hline eVpr & Hi & ب-r-r نفر & \multirow{4}{*}{ تعلداد اعضاى خانواده } \\
\hline $\mathrm{HT/A}$ & r. & |l- انفر & \\
\hline$\cdot$ & - & 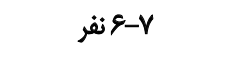 & \\
\hline • & - & 1 و بيشتر & \\
\hline$r+1$. & ir & 1 & \multirow{4}{*}{ تعلاد فرزند } \\
\hline$\pi T / A$ & r. & r & \\
\hline PVR & rq & $r$ & \\
\hline • & - & أو بيشتر & \\
\hline - & • & 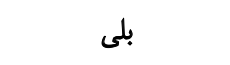 & \multirow{3}{*}{ سابقه مراقبت } \\
\hline $1 .$. & 81 & خير & \\
\hline $1+$. & 91 & جمع كل & \\
\hline
\end{tabular}


جدول r. توزيع فراوانى مطلق و نسبى امتياز بار مراقبتى مادران قبل و بعد از مداخله آموزشى

\begin{tabular}{|c|c|c|c|c|}
\hline \multicolumn{2}{|c|}{ بعد از مداخله } & \multicolumn{2}{|c|}{ قبل از مداخله } & \multirow{2}{*}{ بار مراقبتى } \\
\hline نسبى (درصد) & مطلق & نسبى (درصد) & مطلق & \\
\hline $9 / 1$ & 8 & - & - & Sم) \\
\hline$\Delta V / \Delta$ & ro & $r / T$ & r & متوسط (II - مf) \\
\hline$M / N$ & r. & $V T / N$ & pr & شديد (it-p.e) \\
\hline - & - & $T F / Q$ & 10 & بسيار شديد (1) (AM) \\
\hline $1+$. & 81 & $1 .+$ & 81 & جمع \\
\hline
\end{tabular}

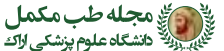

جدول ب. مقايسه ميانكين نمره بار مراقبتى مادران قبل و بعد از مداخله

\begin{tabular}{|c|c|c|c|}
\hline \multicolumn{2}{|c|}{ آزمون تى زوجى } & \multirow{2}{*}{ ميانئين و انحراف نمره بار مراقبتى } & \multirow{2}{*}{ زمان اندازهكيرى } \\
\hline P & $\mathbf{t}$ & & \\
\hline \multirow[t]{2}{*}{$\leq .1 . .1$} & ra/u & & قبل از هداخله \\
\hline & & $R T / \Lambda \pm M / A$ & بعد أز مداخله \\
\hline
\end{tabular}

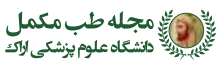

مراقبتى مادران قبل از مداخله رادر سطح متوسط ارزيابى كردند

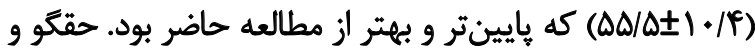

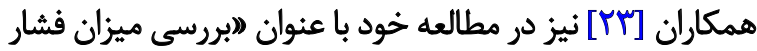

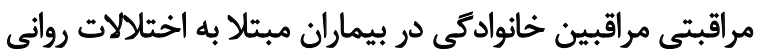

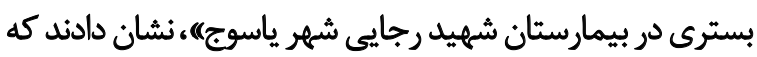

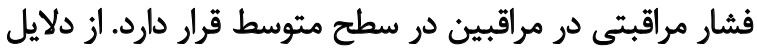

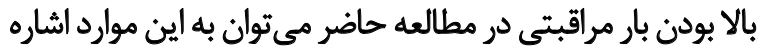

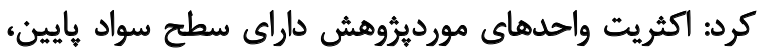

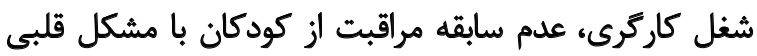

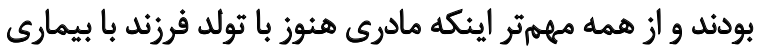

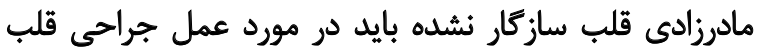

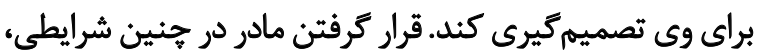

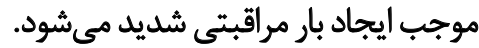

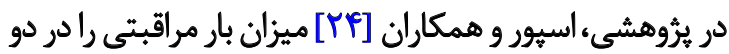

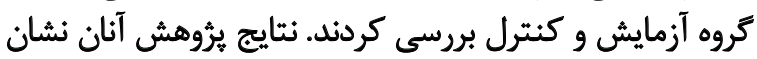

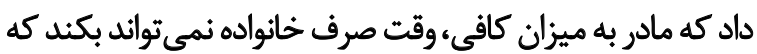

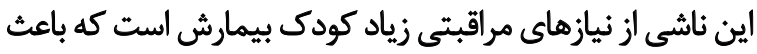

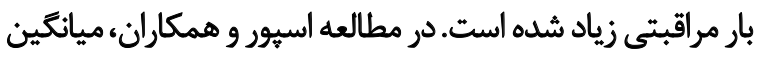

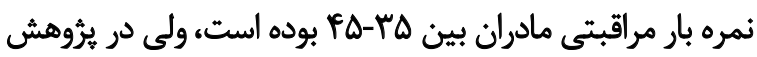

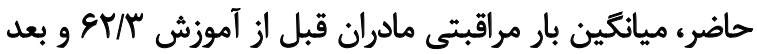

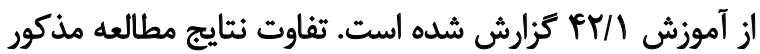

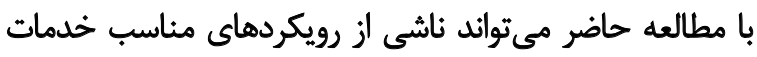

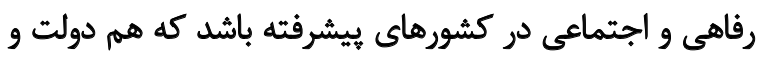

همجنين يافتههاي يُورهش بيانكر آن است كه در مرحله قبل

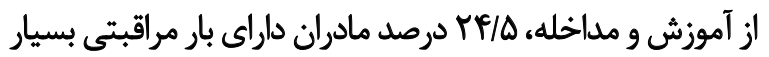

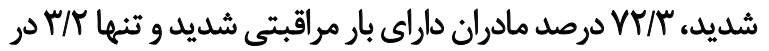

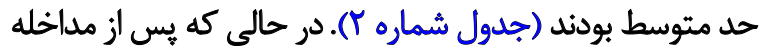

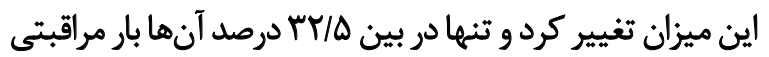

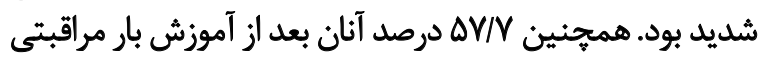
متوسط داشتند (جدول شماره Y). علاوه بر اين ميانكين و انحراف معيار نمره بار مراقبتى مادران

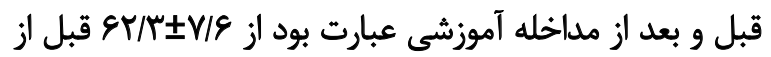

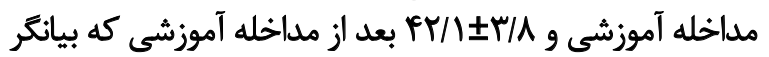

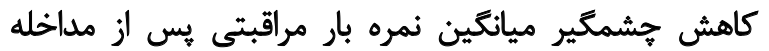

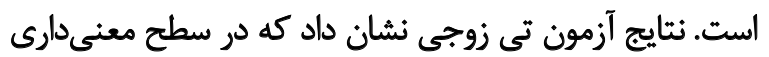

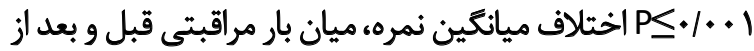

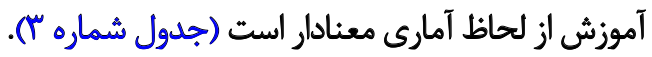

ث๐

مطالعه حاضر با هدف تعيين ميزان بار مراقبتى مادران كودكان

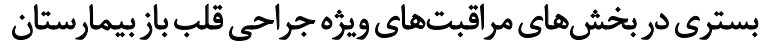

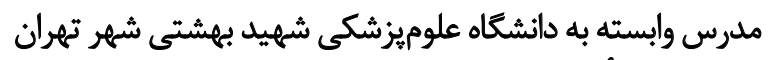

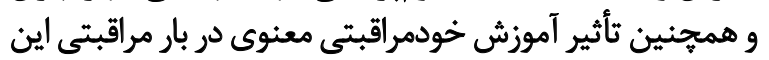

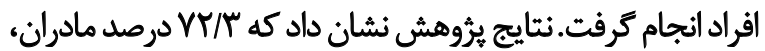

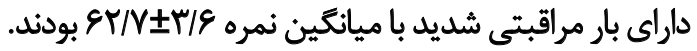
خليفهزاده اصفهانى و همكاران [Tr] در مطالعه خود، بار 
مادران كودكان تحت عمل جراحى قلب در دو كروه آزمايش و

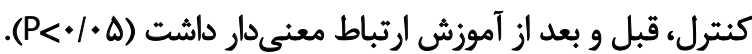

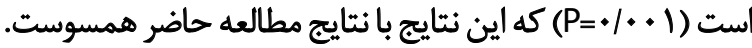

تشابه نتايج مي تواند ناشى از تشابه نوع بيمارى باشد. همجنين

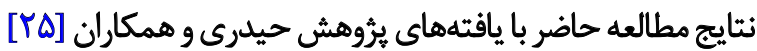

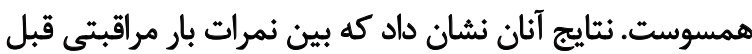

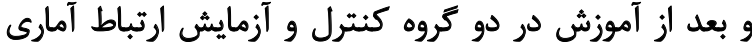

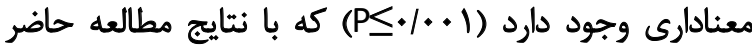

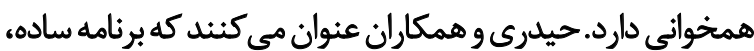

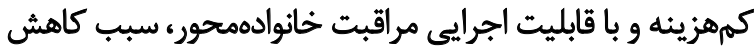

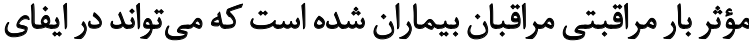

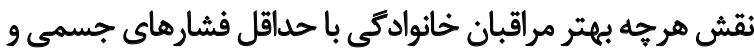

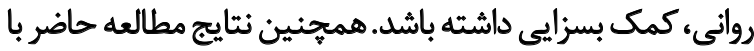
يافتههاى سليمى و همكار ان همسوست. نتيجلميرى فشار مراقبتى مي تواند بر نحوه و كيفيت مراقبتها اثركذار باشدو

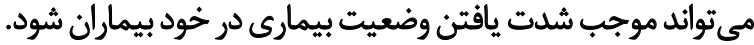

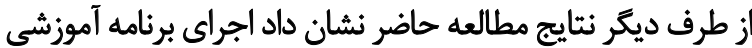

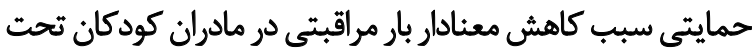

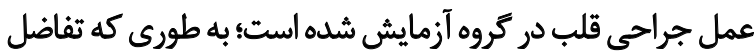

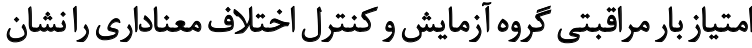

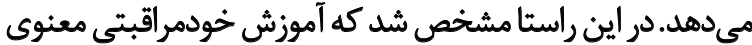

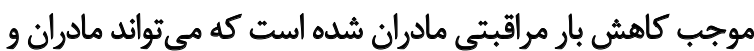

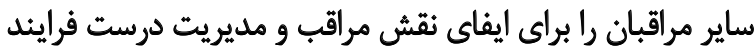

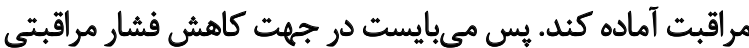

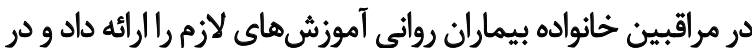

$$
\text { راستاى كاهش آن برنامهريزى كرد. }
$$

مطالعه حاضر نيز هماند ساير مطالعات داراى محدوديتهاى

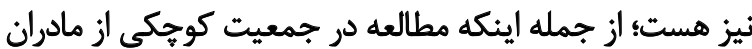

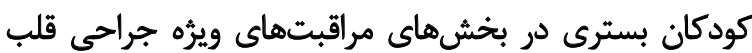

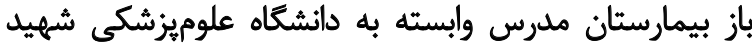

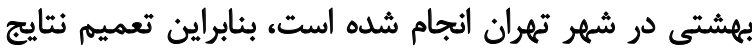

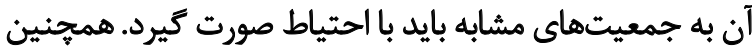

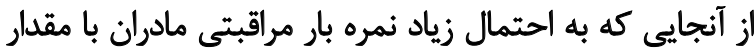

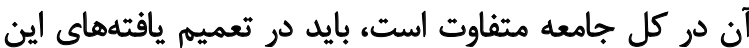

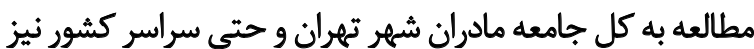

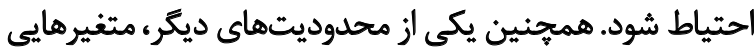
نظير ويثگى هاى فردى مشاركت كنند

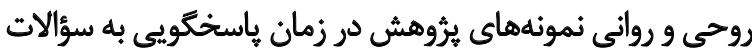

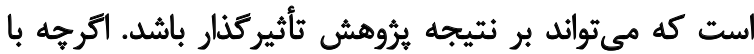

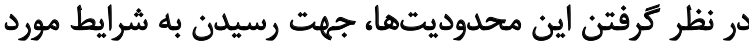

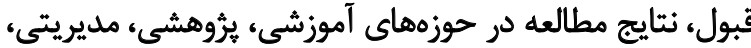

هم خانوادهها در اين شرايط به يارى مادران شتافته و مادر رالز الز

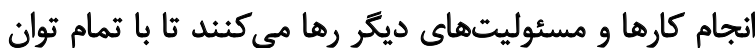

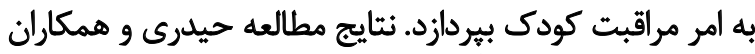

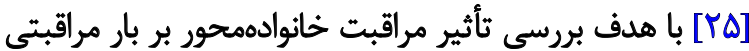

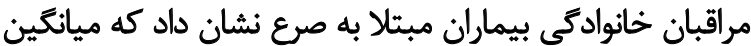

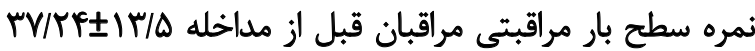

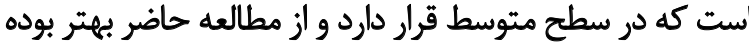

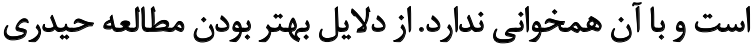

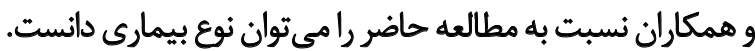

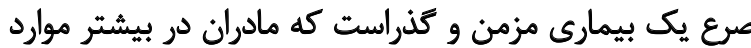

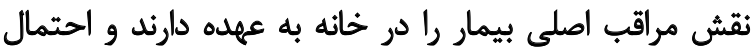

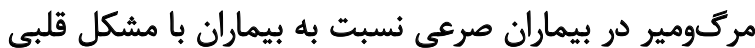

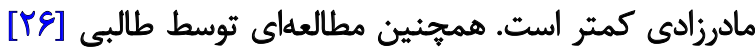

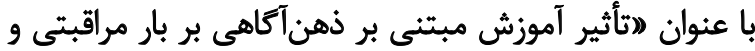

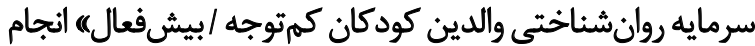

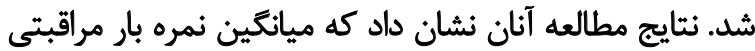

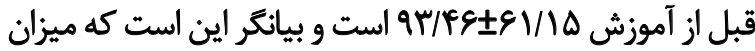
بار مراقبتى مراقبان قبل از مداخله در سطح شديد قرار آدار دارد و با بان مطالعه حاضر همسوست.

يس از مشخص شدن ميزان بار مراقبتى و به منظور بهبيود

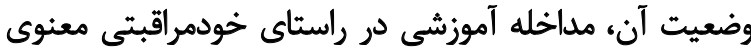

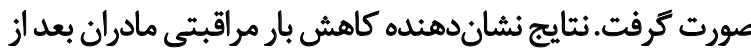

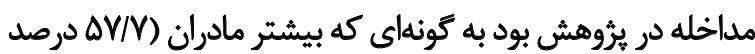

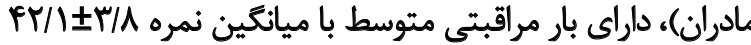

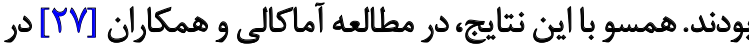

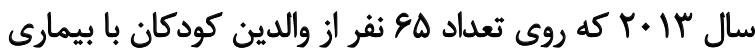

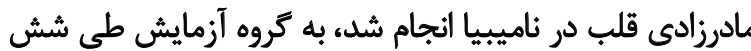

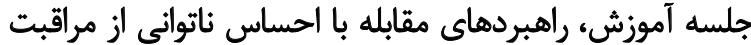

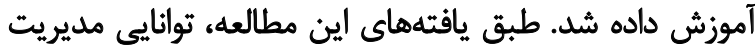

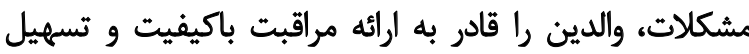

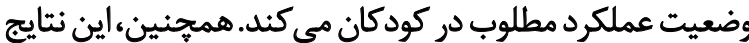

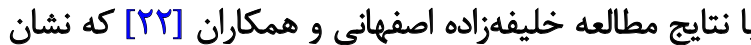

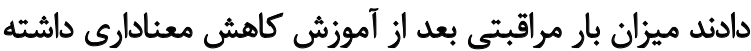

$$
\text { است نيز همسوست. }
$$

ريحاني و همكاران در مطالعه خود به بررسى تأثير آموزش

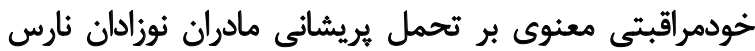

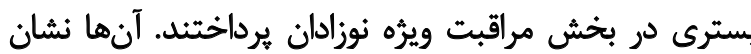

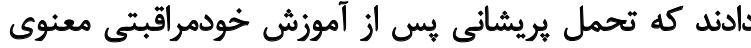

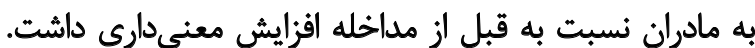

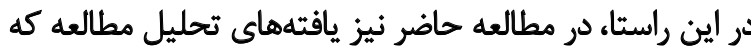

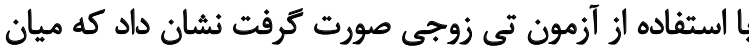

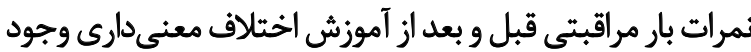

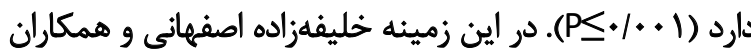

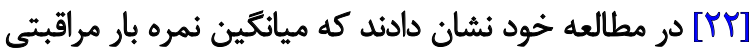


حوزه درمان و ييشكيرى مى تواند كاربرد داشته باشد، اما بيشنهاد

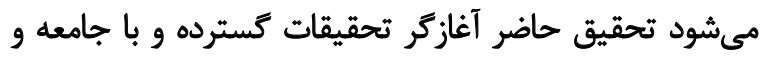

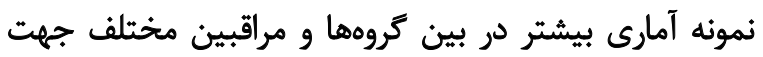
يويايى و رشد دانش برستارى باشد. مالاحظات اخلاقي

$$
\text { ييروى ائز اصول اخلاق يثوهش }
$$

IR.IAU.TMU. اين مطالعه داراى كد اخلاق به شماره

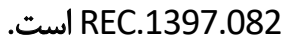

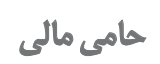

يثروه حاضر بركرفته از إياننامه كارشناسى ارشد نويسنده

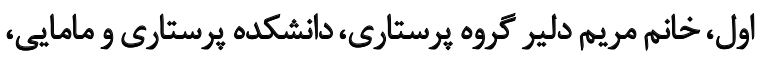

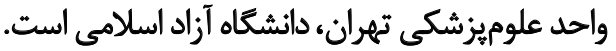

$$
\text { مشاركت نويسندكان }
$$

تمام نويسندكان در نكارش مقاله مشاركت داشتند.

$$
\text { تعارض مناقع }
$$

بنابر اظهار نويسندكان اين مقاله تعارض منافع ندارد.

$$
\text { تشكر و قدردافي }
$$

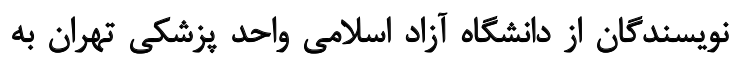

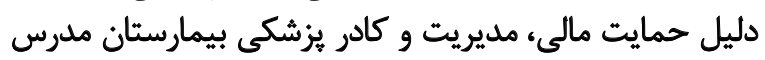

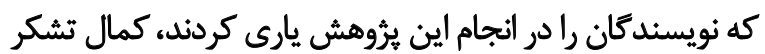
و قدردانى را دارند. 


\section{References}

[1] Keshavars M, Eskandari N, Jahdi F, Ashaieri H, Hoseini F, Kalani M. [The effect of holly Quran recitation on physiological responses of premature infant (Persian)]. Koomesh. 2010; 11(3):169-77. http://koomeshjournal. semums.ac.ir/article-1-730-en.html

[2] Michelena HI, Prakash SK, Della Corte A, Bissell MM, Anavekar N, Mathieu $\mathrm{P}$, et al. Bicuspid aortic valve: Identifying knowledge gaps and rising to the challenge from the International Bicuspid Aortic Valve Consortium (BAVCon). Circulation. 2014; 129(25):2691-704. [DOI:10.1161/ CIRCULATIONAHA.113.007851] [PMID] [PMCID]

[3] Gray PH, Edwards DM, O'Callaghan MJ, Cuskelly M, Gibbons K. Parenting stress in mothers of very preterm infants --influence of development, temperament and maternal depression. Early Human Development. 2013; 89(9):625-9. [DOI:10.1016/j.earlhumdev.2013.04.005] [PMID]

[4] Mohamadi Shahbalaghi F. [Self-efficacy and caregiver strain in Alzheimer's caregivers (Persian)]. Salmand: Iranian Journal of Ageing. 2006; 1(1):26-33. http://salmandj.uswr.ac.ir/article-1-29-en.html

[5] Mastropietro CW, Davalos MC, Seshadri Sh, Walters HL, Delius RE. Clinical response to arginine vasopressin therapy after paediatric cardiac surgery. Cardiology in the Young. 2013; 23(3):387-93. [DOI:10.1017/ S1047951112000996] [PMID]

[6] Nair J, Lakshminrusimha S. Update on PPHN: Mechanisms and treatment. Seminars in Perinatology. 2014; 38(2):78-91. [DOI:10.1053/j.semperi.2013.11.004] [PMID] [PMCID]

[7] Woda A, Belknap RA, Haglund K, Sebern M, Lawrence A. Factors influencing self-care behaviors of African Americans with heart failure: A photovoice project. Heart \& Lung. 2015; 44(1):33-8. [DOI:10.1016/j. hrtlng.2014.09.001] [PMID]

[8] Ku YL. Building spiritual care in nursing: An overview of Ku's spiritual studies in Taiwan. Journal of Christian Nursing. 2017; 34(2):E26-E30. [DOI:10.1097/CNJ.0000000000000377] [PMID]

[9] Navidian A, Bahari F. Burden experienced by family caregivers of patients with mental disorders. Pakistan Journal of Psychological Research. 2008; 23(1-2):19-28. https://www.researchgate.net/publication/277172824

[10] Timmins F, Caldeira S. Understanding spirituality and spiritual care in nursing. Nursing Standard. 2017; 31(22):50-7. [DOI:10.7748/ns.2017. e10311] [PMID]

[11] Büssing A, Waßermann U, Hvidt NC, Längler A, Thiel M. Spiritual needs of mothers with sick new born or premature infants-a cross sectional survey among German mothers. Women and Birth. 2018; 31(2):e89e98. [DOI:10.1016/j.wombi.2017.08.002] [PMID]

[12] Fryer CE, Luker JA, McDonnell MN, Hillier SL. Self management programmes for quality of life in people with stroke. The Cochrane Database of Systematic Reviews. 2016; 2016(8):CD010442. [DOI:10.1002/14651858.CD010442.pub2] [PMID] [PMCID]

[13] Oji VU, Hung LC, Abbasgholizadeh R, Terrell Hamilton F, Essien EJ, Nwulia E. Spiritual care may impact mental health and medication adherence in HIV+ populations. HIV/AIDS (Auckland, NZ). 2017; 9:101-9. [DOI:10.2147/HIV.S126309] [PMID] [PMCID]

[14] Batcheller J, Davis J, Yoder-Wise PS. Hope for the future: Intensifying spirituality in the workplace. Nursing Administration Quarterly. 2013; 37(4):309-16. [DOI:10.1097/NAQ.0b013e3182a2f9ae] [PMID]

[15] Martinez-Rodriguez S, Ortiz-Marqués N, Iraurgi I, Carrasco M, Miguel JJ. Adaptation and analysis of psychometric features of the caregiver risk screen: A tool for detecting the risk of burden in family caregiv- ers. International Psychogeriatrics. 2013; 25(5):755-64 [DOI:10.1017/ S1041610212002426] [PMID]

[16] Chiao CY, Wu HS, Hsiao CY. Caregiver burden for informal caregivers of patients with dementia: A systematic review. International Nursing Review. 2015; 62(3):340-50. [DOI:10.1111/inr.12194] [PMID]

[17] Cheng JF, Huang XY, Lin MJ, Wang YH, Yeh TP. The influence of a mental health home visit service partnership intervention on the caregivers' home visit service satisfaction and care burden. Journal of Clinical Nursing. 2018; 27(3-4):e668-e77. [DOI:10.1111/jocn.14123] [PMID]

[18] Puchalski CM, Vitillo R, Hull SK, Reller N. Improving the spiritual dimension of whole person care: Reaching national and international consensus. Journal of Palliative Medicine. 2014; 17(6):642-56. [DOI:10.1089/ jpm.2014.9427] [PMID] [PMCID]

[19] Bommer C, Heesemann E, Sagalova V, Manne-Goehler J, Atun R, Bärnighausen $\mathrm{T}$, et al. The global economic burden of diabetes in adults aged 20-79 years: A cost-of-illness study. The Lancet Diabetes \& Endocrinology. 2017; 5(6):423-30. [DOI:10.1016/S2213-8587(17)30097-9]

[20] Pahlavanzadeh S, Navidian A, Yazdani M. [The effect of psycho-education on depression, anxiety and stress in family caregivers of patients with mental disorders (Persian)]. Journal of Kermanshah University of Medical Sciences. 2010; 14(3):228-36. https://www.sid.ir/fa/journal/ ViewPaper.aspx?ID=119161

[21] Bagherbeik Tabrizi L, Navab E, Farokhnezhad Afshar P, Asadi Noghab AA, Haghani H. [Effect of cognitive- behavioral intervention on burden of family caregivers of patients with Alzheimer's disease (Persian)]. Hayat. 2015; 21(1):94-102. http://hayat.tums.ac.ir/article-1-1058-en.htm

[22] Khalifehzadeh A, Gheisarian Z, Bigdelian $\mathrm{H}$. [The effect of care program on care giving burden of mothers of children undergoing congenita heart disease surgery (Persian)]. Iranian Journal of Pediatric Nursing. 2018; 4(3):41-6. http://jpen.ir/article-1-266-en.html

[23] Haghgoo A, Zoladl M, Afroughi S, Rahimian H, Mirzaee MS. [Assessment of the burden on family caregivers of patients with mental disorders hospitalized in Shahid Rajai Hospital in Yasuj, 2016 (Persian)] Iranian Journal of Psychiatric Nursing. 2017; 5(2):39-44. [In Persian] [DOI:10.21859/ijpn-05026]

[24] Spore E. The quality of life primary caregivers of children with chronic conditions [PhD. dissertation]. Chicago: University of Illinois at Chicago; 2012. https://indigo.uic.edu/articles/The_Quality_of_Life_of_Caregivers_of_Children_with_Chronic_Conditions/10809086/files/19319504. pdf

[25] Heidari M, Etemadi Far Sh, Masoudi R, Kheyri S, Jivad N. [The effect of family-centered care on the family caregivers' burden of patients with epilepsy (Persian)]. Journal of Clinical Nursing and Midwifery. 2018; 7(2):146-57. http://jcnm.skums.ac.ir/article-1-768-en.html

[26] Talebi S. [The effect of mindfulness-based education on the burden of care and psychological capital of parents of children with ADHD (Persian)] [MSc. thesis]. Sari: Mazandaran University of Medical Sciences; 2018. http://eprints.mazums.ac.ir/id/eprint/4590

[27] Amakali K, Small LF. Empowerment of the rural parents/caregivers of children with heart diseases in Namibia to facilitate coping with the demands of caring at home. Global Journal of Health Science. 2013; 5(2):74-83. [DOl:10.5539/gjhs.v5n2p74] 
This Page Intentionally Left Blank 